Probability, Networks and Algorithms

Probability, Networks and Algorithms

PNA Packet models revisited: tandem and priority systems

M.R.H. Mandies

Report PNA-E0405 June 2004 
CWI is the National Research Institute for Mathematics and Computer Science. It is sponsored by the Netherlands Organization for Scientific Research (NWO).

$\mathrm{CWI}$ is a founding member of ERCIM, the European Research Consortium for Informatics and Mathematics.

CWI's research has a theme-oriented structure and is grouped into four clusters. Listed below are the names of the clusters and in parentheses their acronyms.

\section{Probability, Networks and Algorithms (PNA)}

Software Engineering (SEN)

Modelling, Analysis and Simulation (MAS)

Information Systems (INS)

Copyright (C) 2004, Stichting Centrum voor Wiskunde en Informatica

P.O. Box 94079, 1090 GB Amsterdam (NL)

Kruislaan 413, 1098 SJ Amsterdam (NL)

Telephone +31205929333

Telefax +31205924199

ISSN 1386-3711 


\title{
Packet models revisited: tandem and priority systems
}

\begin{abstract}
We examine two extensions of traditional single-node packet-scale queueing models: tandem networks and (strict) priority systems. Two generic input processes are considered: periodic and Poisson arrivals. For the two-node tandem, an exact expression is derived for the joint distribution of the total queue length, and the queue length of the first queue, implicitly determining the distribution of the second queue. Similarly we derive the distribution of the lowpriority queue in a two-class priority system. We also provide explicit approximations based on the Brownian bridge.
\end{abstract}

2000 Mathematics Subject Classification: 60K25

Keywords and Phrases: Queueing, packet models, periodic and Poisson input, tandem systems, priority systems, Brownian bridge

Note: Part of this work was carried out in the project EQUANET, supported by the Dutch Department of Economic Affairs/SENTER 


\title{
Packet models revisited: tandem and priority systems
}

\section{Michel MANDJES ${ }^{1}$}

\author{
CWI Department of Applied Mathematics \\ Advanced Communication Networks University of Twente \\ $\begin{array}{ll}\text { P.O. Box } 94079 & \text { P.O. Box } 217\end{array}$ \\ 1090 GB Amsterdam \\ 7500 AE Enschede \\ the Netherlands \\ the Netherlands
}

\begin{abstract}
We examine two extensions of traditional single-node packetscale queueing models: tandem networks and (strict) priority systems. Two generic input processes are considered: periodic and Poisson arrivals. For the two-node tandem, an exact expression is derived for the joint distribution of the total queue length, and the queue length of the first queue, implicitly determining the distribution of the second queue. Similarly we derive the distribution of the low-priority queue in a two-class priority system. We also provide explicit approximations based on the Brownian bridge.
\end{abstract}

Keywords Queueing $\bullet$ packet models $\bullet$ periodic and Poisson input $\bullet$ tandem systems $\bullet$ priority systems $\bullet$ Brownian bridge

\footnotetext{
${ }^{1}$ Part of this work was carried out in the project EQUANET, supported by the Dutch Department of Economic Affairs/SENTER. Email: michel@cwi.nl.
} 


\section{INTRODUCTION}

Packet-level models are used to describe congestion phenomena in communication networks, particularly those related to relatively short time-scales. On this time-scale (typically in the ms-range), the network element (switch, router) has a (nearly) constant number of users, each transmitting packets at a constant rate. This resulted in the canonical periodic traffic model: $N$ users transmit a packet every $D$ units of time, in a purely periodic fashion. When $N$ is large, this is accurately approximated by a Poisson process with rate $\lambda \equiv N / D$.

The above motivates the intensive examination of two generic packet-level single-node queueing models: $N \cdot \mathrm{D} / \mathrm{D} / 1$ ( $N$ periodic arrivals) and $\mathrm{M} / \mathrm{D} / 1$ (Poisson arrivals), where the deterministic service is justified by the fact that in communication networks packet service times are constant.

Single queue. The solution of the workload distribution in the $N \cdot \mathrm{D} / \mathrm{D} / 1$ queue goes back to Dempster [4], Pyke [12], and Takács [19], who independently found elegant explicit expressions. Takacs's approach is based on combinatorial arguments, e.g., Ballot theorems; the translation of this result into the $N \cdot \mathrm{D} / \mathrm{D} / 1$ context is due to Humblet et al. [8], see also [2]. Eckberg [5], apparently not aware of the explicit results, found a recursive algorithm for computing the distribution function of the workload; see for another exact derivation also [6]. Independently of [8] (and nearly simultaneously), Virtamo and Roberts [14, 22] rederived Takács' closed form solution; Norros et al. [11] noted that the approach followed in $[14,22]$ can be viewed as an application of the Beneš method [1]. In [7, 11, 16] Brownian-bridge approximations are proposed, which are particularly accurate in a heavy-traffic environment. A concise survey on the single $N \cdot \mathrm{D} / \mathrm{D} / 1$ queue is found in [13, Section 15.2]. The $\mathrm{M} / \mathrm{D} / 1$ waiting line being a special case of $\mathrm{M} / \mathrm{G} / 1$, its history goes back to the times of Erlang. The Laplace transform of the workload is obtained directly from the Pollaczek-Khinchin formula. Explicit formulas for the distribution function are available as well, though some care is required to avoid numerical instabilities. A short survey on this issue, including a number of accurate approximations, is given in [13, Section 15.1]. 
Beyond the single queue. The single-node model obviously gives valuable insights, but is an oversimplification of reality. We mention two serious limitations. First, traffic streams usually traverse concatenations of hops (rather than just a single node). Secondly, it is envisaged that at the network nodes service differentiation is implemented (for instance by using priority mechanisms). This motivates the interest in performance evaluation for these more complex queueing models.

It is noted that the traffic models (periodic and Poisson arrivals) described above are of a generic nature, and consequently not limited to a specific network technology. In [13] they are placed in the framework of performance evaluation in ATM networks, but they are appropriate for delay assessments in IP-based networks as well, see e.g. [9, 10, 23].

Two desirable extensions of the single-node model are the tandem and priority queue. For the model with Poissonian input results have been available for a long time, see e.g. Takagi [20]. For periodic input, however, results are less satisfactory.

Virtamo [21] considers the distribution of the busy and idle periods of the $N \cdot \mathrm{D} / \mathrm{D} / 1$ queue. The main motivation of this study lies in describing the output process of a node, to be able to analyze concatenations of nodes. Here it is noted that the output of the queue is an on-off process; busy (idle) periods of the queue correspond to on-times (off-times, respectively). However, remark that consecutive busy (and idle) periods are not independent. Consider for instance, in the situation of a queue with link rate $c$ and sources with period $D$, a busy period of length $N / c$; then necessarily the next idle period has length $D-N / c$, etc. This implies that the (marginal) distributions of the busy and idle periods do not describe unambiguously the output process.

A complete probabilistic description of the output process of the $N \cdot \mathrm{D} / \mathrm{D} / 1$ queue is given by Boyer et al. [3]. This could, in principle, be used to analyze the two-node tandem. However, the description is rather complex, and does not lend itself to explicit analysis.

Contribution $\&$ organization. In this paper we derive closed-form expressions for the distribution function of the workload in the tandem and priority queue, 
both with periodic and Poisson arrivals. More specifically, the contributions of our work include:

- An explicit solution of the joint distribution of the workloads of the queues. This is done for both the two-node tandem queue (Section 2), and the two-class priority queue (Section 3). To our best knowledge, for periodic arrivals these expressions were not known so far. For Poisson arrivals, a vast body of literature is available, but a considerable part of it is in terms of Laplace transforms (see, e.g., [15, 17, 20]), whereas our solution is explicit.

- Brownian-bridge approximations for both periodic and Poisson input (Section 4). The use of these approximations is illustrated by a numerical example.

\section{TANDEM SYSTEM}

This section is devoted to the exact analysis of tandem network, both for periodic and Poisson arrivals. We start by presenting some preliminaries.

\subsection{Preliminaries}

In a tandem network, the output of a first queue is fed into a second queue. We consider queues with constant service rates, say, $c_{1}$ and $c_{2}$, respectively. To avoid trivialities, we assume that (i) $c_{1}>c_{2}$ (otherwise the second queue remains empty), (ii) the mean amount of traffic offered to the network per unit of time is smaller than $c_{2}$. Denoting by $A(s, t)$ the amount of traffic generated in the interval $[s, t)$ (for $s<t$ ), Assumption (ii) reduces to $\mathbb{E} A(0,1)=: \rho<c_{2}$. Denote the steady-state content of queue $i$ by $Q_{i}$, and the steady-state content of the entire network by $\bar{Q}$. The classical Reich formula gives, for the single queue, the relation between the steady-state buffer content and the arrival process. Evidently, this formula applies to queue $1: Q_{1} \stackrel{\mathrm{d}}{=} \sup _{t \geq 0}(A(-t, 0)-$ $\left.c_{1} t\right)$. Little thought gives that, assuming that traffic leaves the first queue as fluid, the total queue $\bar{Q}$ behaves as a single queue emptied at rate $c_{2}$. Consequently we also have that $\bar{Q} \stackrel{\mathrm{d}}{=} \sup _{t \geq 0}\left(A(-t, 0)-c_{2} t\right)$. 
The goal of this paper is to analyze the workload distribution of the second queue. Evidently, this probability can be derived from

$$
\mathbb{P}\left(Q_{1} \leq x_{1}, \bar{Q} \leq x_{2}\right)=\mathbb{P}\left(\forall t \geq 0: A(-t, 0) \leq \min \left\{x_{1}+c_{1} t, x_{2}+c_{2} t\right\}\right),
$$

with $x_{1}<x_{2}$, as follows:

$$
\mathbb{P}\left(Q_{2} \leq y\right)=\int_{0}^{\infty}\left(\left.\frac{\partial}{\partial x_{1}} \mathbb{P}\left(Q_{1} \leq x_{1}, \bar{Q} \leq x_{2}\right)\right|_{x_{1}:=x, x_{2}:=x+y}\right) \mathrm{d} x .
$$

Notice that, due to time-reversibility arguments, we can replace $A(-t, 0)$ in the above formulae by $A(0, t)$.

\subsection{Periodic arrivals}

Consider the sequence $T_{1}, \ldots, T_{N}$ of i.i.d. random variables that are distributed uniformly on $[0, D)$. For ease, we set $A(t):=A(0, t)$. Then $A(t)$ is the number of these events that has occurred before $t$, i.e.,

$$
A(t)=\#\left\{i: T_{i} \leq t\right\} .
$$

Define

$$
p(x, c \mid N, D):=\mathbb{P}(\forall t \in[0, D): A(t) \leq x+c t) .
$$

For ease of notation, we also define binomial probabilities, as follows:

$$
\mathbb{B i n}(n \mid N, p):=\left(\begin{array}{l}
N \\
n
\end{array}\right) p^{n}(1-p)^{N-n} .
$$

The following lemma is a direct consequence of earlier results. Observe that it, in particular, implies that $p(x, c \mid N, D)=1$ if $x \geq N$, as desired.

Lemma 2.1 For $x \in[0, N]$ and $N<x+c \cdot D$, it holds that

$$
p(x, c \mid N, D)=1-\sum_{n \in \mathbb{N}: n \in(x, N]} \mathbb{B i n}\left(n \mid N, \frac{n-x}{c \cdot D}\right) \cdot \frac{c \cdot D-N+x}{c \cdot D-n+x} .
$$

If $N \geq x+c \cdot D$, then $p(x, c \mid N, D)=0$. 


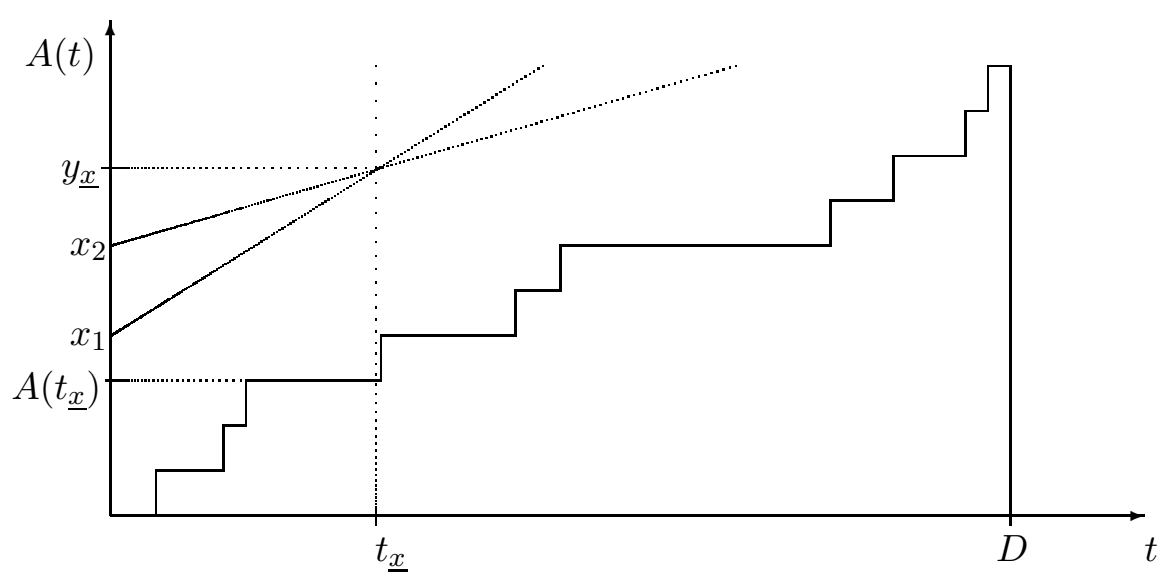

Figure 1: Example for the proof of Proposition 2.2. The parameters used are $N=10, D=20 ;$ in this scenario $A\left(t_{\underline{x}}\right)=3$.

Proof. This is proven in Humblet et al. [8, Section III.C], and Roberts and Virtamo [14].

Now fix two intercepts $x_{1}, x_{2}$ and two slopes $c_{1}, c_{2}$. Define $\left(t_{\underline{x}}, y_{\underline{x}}\right)$ as the intersection of the lines $x_{1}+c_{1} t$ and $x_{2}+c_{2} t$, i.e.,

$$
t_{\underline{x}}=\frac{x_{2}-x_{1}}{c_{1}-c_{2}}, \quad y_{\underline{x}}=\frac{c_{1} x_{2}-c_{2} x_{1}}{c_{1}-c_{2}} .
$$

Note that under $x_{1}<x_{2}$ and $c_{1}>c_{2}$, both $t_{\underline{x}}$ and $y_{\underline{x}}$ are positive.

Now define the 'two-dimensional analog' of $p(x, c, \mid N, D)$ :

$$
p_{\mathrm{t}}(\underline{x}, \underline{c} \mid N, D):=\mathbb{P}\left(\forall t \in[0, D): A(t) \leq \min \left\{x_{1}+c_{1} t, x_{2}+c_{2} t\right\}\right) .
$$

Notice that (reverse time!) this probability coincides with our target probability (1).

Proposition 2.2 Fix $0<x_{1}<x_{2}$ and $N<c_{2} \cdot D$ (with $c_{1}>c_{2}$ ). If $t_{\underline{x}}>D$, then

$$
\begin{aligned}
& p_{\mathrm{t}}(\underline{x}, \underline{c} \mid N, D)=p\left(x_{1}, c_{1} \mid N, D\right) . \\
& \text { If } t_{\underline{x}} \leq D, \text { then } \\
& p_{\mathrm{t}}(\underline{x}, \underline{c} \mid N, D)=\sum_{n=0}^{\min \left\{\left\lfloor y_{\underline{x}}\right\rfloor, N\right\}} \mathbb{B i n}\left(n \mid N, \frac{t_{\underline{x}}}{D}\right) \\
& \quad \cdot p\left(x_{1}, c_{1} \mid n, t_{\underline{x}}\right) \cdot p\left(y_{\underline{x}}-n, c_{2} \mid N-n, D-t_{\underline{x}}\right) .
\end{aligned}
$$


Proof. The first claim is obvious, as the $x_{i}+c_{i} t$ do not intersect before $D$. Now consider the second claim. Partition to the feasible values of $A\left(t_{\underline{x}}\right)$, see Figure 1. Clearly,

$$
\mathbb{P}\left(A\left(t_{\underline{x}}\right)=n\right)=\mathbb{B i n}\left(n \mid N, \frac{t_{\underline{x}}}{D}\right) .
$$

Conditional on this event, we have, independently of each other, $n$ i.i.d. arrivals, distributed uniformly on $\left[0, t_{\underline{x}}\right]$, and $N-n$ i.i.d. arrivals, distributed uniformly on $\left[t_{\underline{x}}, D\right)$. It is now straightforward to derive (2).

\subsection{Poisson arrivals}

Consider a sequence $\left(S_{i}\right)_{i \in \mathbb{N}}$ of i.i.d. random variables that are exponentially distributed with mean $\lambda^{-1}$. Define $T_{i}:=\sum_{j=1}^{i} S_{j}$. As before:

$$
\begin{aligned}
& q(x, c \mid \lambda):=\mathbb{P}(\forall t \in[0, \infty): A(t) \leq x+c t), \quad \text { and } \\
& q_{\mathrm{t}}(\underline{x}, \underline{c} \mid \lambda):=\mathbb{P}\left(\forall t \in[0, \infty): A(t) \leq \min \left\{x_{1}+c_{1} t, x_{2}+c_{2} t\right\}\right) ;
\end{aligned}
$$

here $q_{\mathrm{t}}(\underline{x}, \underline{c} \mid \lambda)$ corresponds to our target probability (1). For ease of notation, we also define Poisson probabilities, as follows:

$$
\operatorname{Pois}(n \mid \lambda):=e^{-\lambda} \frac{\lambda^{n}}{n !} \text {. }
$$

Lemma 2.3 is a standard result for the M/D/1 queue, see e.g. [13, Eq. (5.1.13)]. The proof of Proposition 2.4 is along the same lines as the proof of Proposition 2.2.

Lemma 2.3 For $x>0$ and $\rho:=\lambda / c<1$, it holds that

$$
q(x, c \mid \lambda)=1-(1-\rho) \sum_{n=\lceil x\rceil}^{\infty} \operatorname{Pois}(n \mid \rho(n-x)) .
$$

Proposition 2.4 Fix $0<x_{1}<x_{2}$ and $c_{1}>c_{2}>\lambda$. Then

$$
q_{\mathrm{t}}(\underline{x}, \underline{c} \mid \lambda)=\sum_{n=0}^{\left\lfloor y_{\underline{x}}\right\rfloor} \operatorname{Pois}\left(n \mid \lambda t_{\underline{x}}\right) \cdot p\left(x_{1}, c_{1} \mid n, t_{\underline{x}}\right) \cdot q\left(y_{\underline{x}}-n, c_{2} \mid \lambda\right) .
$$




\section{PRIORITY SYSTEM}

In this section we address the priority queue, both for periodic and Poisson arrivals. The analysis is more involved than that of the tandem queue.

\subsection{Prelimaries}

Consider a (strict) priority system, fed by two traffic streams. Traffic of type $i$ is led into queue $i, i=1,2$. Stream 1 is served with priority, whereas stream 2 is only served when queue 1 is empty. With notation analogously to the tandem case, and $\bar{A}(s, t):=A_{1}(s, t)+A_{2}(s, t)$ for $s<t$, stability is guaranteed if $A_{1}(0,1)+A_{2}(0,1)<c$.

Again, denote the steady state content of queue $i$ by $Q_{i}$, and the steady-state content of the entire network by $\bar{Q}$. Because class 1 does not 'see' class 2 , $Q_{1} \stackrel{\mathrm{d}}{=} \sup _{t \geq 0} A_{1}(-t, 0)-c t$. However, as the total system is work-conserving, we also have $\bar{Q}$ is distributed as $\sup _{t \geq 0} \bar{A}(-t, 0)-c t$. This mean that, as before, $\mathbb{P}\left(Q_{1} \leq x_{1}, \bar{Q} \leq x_{2}\right)$ can be rewritten as

$$
\mathbb{P}\left(\forall t \geq 0:\left\{A_{1}(-t, 0) \leq x_{1}+c t\right\} \cap\left\{\bar{A}(-t, 0) \leq x_{2}+c t\right\}\right),
$$

with $x_{1}<x_{2}$.

\subsection{Periodic arrivals}

Now consider $N_{i} \in \mathbb{N}$ arrivals of type $i$ in the interval $[0, D)$, for $i=1,2$. It is straightforward that probability (4) reads

$$
p_{\mathrm{p}}(\underline{x}, c \mid \underline{N}, D):=\mathbb{P}\left(\forall t \in[0, D):\left\{A_{1}(t) \leq x_{1}+c t\right\} \cap\left\{\bar{A}(t) \leq x_{2}+c t\right\}\right) .
$$

This probability is calculated in four steps.

- We first observe

$$
p_{\mathrm{p}}(\underline{x}, c \mid \underline{N}, D)=p\left(x_{1}, c \mid N_{1}, D\right)-\bar{p}_{\mathrm{p}}(\underline{x}, c \mid \underline{N}, D)
$$

with $\bar{p}_{\mathrm{p}}(\underline{x}, c \mid \underline{N}, D):=\mathbb{P}(E)$, with event $E$ defined as

$$
\left\{\forall t \in[0, D): A_{1}(t) \leq x_{1}+c t ; \exists t \in[0, D): \bar{A}(t)>x_{2}+c t\right\} .
$$




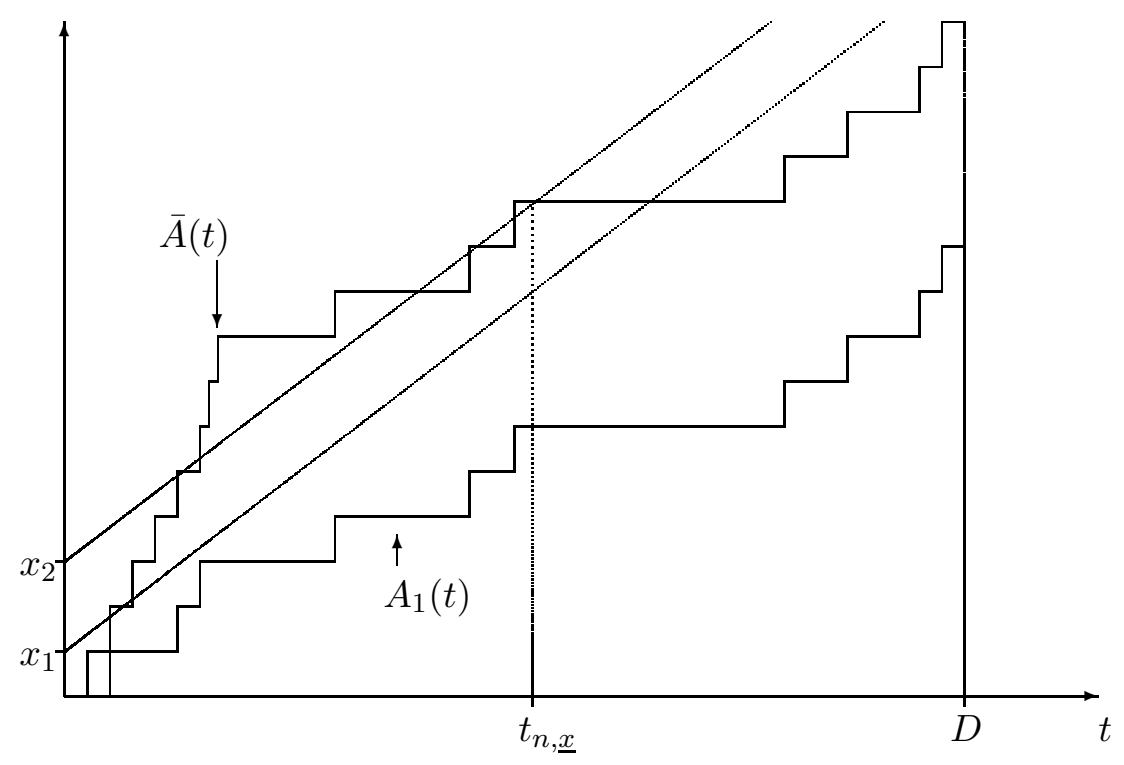

Figure 2: Example for the proof of Theorem 3.1. The parameters used are $N_{1}=10, N_{2}=5, D=20$; in this scenario $\bar{A}\left(t_{n, \underline{x}}\right)=11$.

- Partition $E$ with respect to the last epoch before $D$ at which $\bar{A}(t) \geq$ $x_{2}+c t$. Let $E(n)$ denote the event

$$
\left\{\begin{array}{l}
\forall t \in[0, D): A_{1}(t) \leq x_{1}+c t \\
\bar{A}\left(t_{n, \underline{x}}\right)=n, \quad \forall t \in\left[t_{n, \underline{x}}, D\right): \bar{A}\left(t_{n, \underline{x}}, t\right) \leq x_{2}-n+c t
\end{array}\right\},
$$

with time epoch $t_{n, \underline{x}}$ is defined as $\left(n-x_{2}\right) / c$. Because the resulting 'subevents' $E(n)$ are disjoint, we get the sum

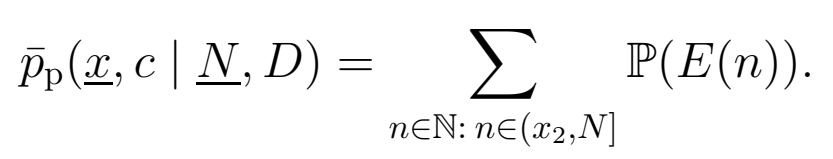

Notice that this approach is essentially equivalent to Beneš's, see [1] and also Equation (2.4) in [11]. As $\left\{\bar{A}\left(t_{n, \underline{x}}, t\right) \leq x_{2}-n+c t\right\}$ implies $\left\{A_{1}\left(t_{n, \underline{x}}, t\right) \leq x_{2}-n+c t\right\}$, the event $E(n)$ equals

$$
\left\{\begin{array}{l}
\forall t \in\left[0, t_{n, \underline{x}}\right): A_{1}(t) \leq x_{1}+c t \\
\bar{A}\left(t_{n, \underline{x}}\right)=n, \quad \forall t \in\left[t_{n, \underline{x}}, D\right): \bar{A}\left(t_{n, \underline{x}}, t\right) \leq x_{2}-n+c t
\end{array}\right\} .
$$

- Now notice that

$$
\mathbb{P}\left(\bar{A}\left(t_{n, \underline{x}}\right)=n\right)=\mathbb{B i n}\left(n \mid N_{1}+N_{2}, \frac{t_{n, \underline{x}}}{D}\right) .
$$


It is a straightforward exercise to show that

$$
\mathbb{P}\left(A_{1}\left(t_{n, \underline{x}}\right)=i \mid \bar{A}\left(t_{n, \underline{x}}\right)=n\right)=\mathbb{H g}\left(i \mid N_{1}, N_{2}, n\right),
$$

for $i \in\left\{0, i^{\star}\right\}$, where $i^{\star}:=\min \left\{n, N_{1}\right\}$, and the hypergeometric distribution is defined by

$$
\mathbb{H g}(m \mid M, N, n):=\left(\begin{array}{c}
M \\
m
\end{array}\right)\left(\begin{array}{c}
N \\
n-m
\end{array}\right) /\left(\begin{array}{c}
M+N \\
n
\end{array}\right) .
$$

- It is evident that, conditional on $\left\{A_{1}\left(t_{n, \underline{x}}\right)=i\right\} \cap\left\{\bar{A}\left(t_{n, \underline{x}}\right)=n\right\}$, there is independence between the events

$$
\begin{aligned}
& \left\{\forall t \in\left[0, t_{n, \underline{x}}\right): A_{1}(t) \leq x_{1}+c t\right\} \text { and } \\
& \left\{\forall t \in\left[t_{n, \underline{x}}, D\right): \bar{A}\left(t_{n, \underline{x}}, t\right) \leq x_{2}-n+c t\right\} .
\end{aligned}
$$

The corresponding conditional probabilities are $p\left(x_{1}, c \mid i, t_{n, \underline{x}}\right)$ and

$$
p\left(0, c \mid N_{1}+N_{2}-n, D-t_{n, \underline{x}}\right)=1-\frac{N_{1}+N_{2}-n}{c\left(D-t_{n, \underline{x}}\right)},
$$

respectively.

This results in the following theorem.

Theorem 3.1 Fix $0<x_{1}<x_{2}$ and $N<c \cdot D$. Then

$$
\begin{gathered}
p_{\mathrm{p}}(\underline{x} \mid \underline{N}, D)=p\left(x_{1}, c \mid N_{1}, D\right)-\sum_{n \in \mathbb{N}: n \in\left(x_{2}, N\right]} \mathbb{B i n}\left(n \mid N_{1}+N_{2}, \frac{n-x_{2}}{c \cdot D}\right) . \\
\sum_{i=0}^{i^{\star}} \mathbb{H} g\left(i \mid N_{1}, N_{2}, n\right) \cdot p\left(x_{1}, c \mid i, t_{n, \underline{x}}\right) \cdot\left(1-\frac{N_{1}+N_{2}-n}{c\left(D-t_{n, \underline{x}}\right)}\right) .
\end{gathered}
$$




\subsection{Poisson arrivals}

Consider a Poisson stream of rate $\lambda_{i}$ for class $i$, for $i=1,2$. Denote $\bar{\lambda}:=\lambda_{1}+\lambda_{2}$. Assume $\bar{\lambda}<c$, to ensure stability. Define

$$
q_{\mathrm{p}}(\underline{x}, c \mid \lambda):=\mathbb{P}\left(\forall t \in[0, \infty):\left\{A_{1}(t) \leq x_{1}+c t\right\} \cap\left\{\bar{A}(t) \leq x_{2}+c t\right\}\right) .
$$

We use the same steps as before:

$$
\begin{aligned}
& \mathbb{P}\left(\bar{A}\left(t_{n, \underline{x}}\right)=n\right)=\mathbb{P} \text { ois }\left(n \mid \bar{\lambda} t_{n, \underline{x}}\right) ; \\
& \mathbb{P}\left(A_{1}\left(t_{n, \underline{x}}\right)=i \mid \bar{A}\left(t_{n, \underline{x}}\right)=n\right)=\mathbb{B i n}\left(i \mid n, \frac{\lambda_{1}}{\bar{\lambda}}\right) .
\end{aligned}
$$

Now consider the events (5) and (6), with the time interval in the latter replaced by $\left[t_{n, \underline{x}}, \infty\right)$. Again, conditional on $\left\{A_{1}\left(t_{n, \underline{x}}\right)=i\right\} \cap\left\{\bar{A}\left(t_{n, \underline{x}}\right)=n\right\}$, these events are independent, and their (conditional) probabilities are given by $p\left(x_{1}, c \mid i, t_{n, \underline{x}}\right)$ and $1-\bar{\lambda} / c$, respectively.

Proposition 3.2 Fix $0<x_{1}<x_{2}$ and $\bar{\lambda}<c$. Then

$$
\begin{gathered}
q_{\mathrm{p}}(\underline{x}, c \mid \underline{\lambda})=q\left(x_{1}, c \mid \lambda_{1}\right)-\sum_{n \in \mathbb{N}: n \in\left(x_{2}, N\right]} \mathbb{P o i s}\left(n \mid \bar{\lambda} t_{n, \underline{x}}\right) . \\
\sum_{i=0}^{n} \mathbb{B} \operatorname{in}\left(i \mid n, \frac{\lambda_{1}}{\bar{\lambda}}\right) \cdot p\left(x_{1}, c \mid i, t_{n, \underline{x}}\right) \cdot\left(1-\frac{\bar{\lambda}}{c}\right) .
\end{gathered}
$$

\section{APPROXIMATIONS, NUMERICAL RESULTS}

In this section we present explicit approximations of the formulae of Section 2; a similar approach could be pursued for the priority case. We illustrate the approximations with an example.

\subsection{Approximations for the tandem queue with periodic arrivals}

Let $\bar{B}(\cdot)$ a Brownian bridge, i.e., a standard Brownian motion $B(\cdot)$ conditioned on $B(1)=0$. A classical result is that

$$
p_{\mathrm{BB}}(x, c):=\mathbb{P}(\forall t \in[0,1): \bar{B}(t) \leq x+c t)=1-p_{\mathrm{BB}}^{-}(x, c),
$$


with $p_{\overline{\mathrm{BB}}}(x, c):=\exp (-2 x(x+c))$,

under the proviso that $x+c \geq 0$, see for instance [18, Eq. (2.2.8)]. It is straightforward to derive that

$$
\begin{aligned}
\mathbb{P} & (\forall t \in[0, T): B(t) \leq x+c t \mid B(T)=z)= \\
& =\mathbb{P}(\forall s \in[0,1): B(s T) \leq x+c s T \mid B(T)=z) \\
& =\mathbb{P}(\forall s \in[0,1): \sqrt{T} B(s) \leq x+c s T \mid \sqrt{T} B(1)=z) \\
& =\mathbb{P}\left(\forall s \in[0,1): B(s) \leq \frac{x}{\sqrt{T}}+\left(c \sqrt{T}-\frac{z}{\sqrt{T}}\right) s \mid B(1)=0\right) \\
& =p_{\mathrm{BB}}\left(\frac{x}{\sqrt{T}}, c \sqrt{T}-\frac{z}{\sqrt{T}}\right) .
\end{aligned}
$$

In this subsection we concentrate on

$$
p_{\mathrm{BB}}(\underline{x}, \underline{c}):=\mathbb{P}\left(\forall t \in[0,1): \bar{B}(t) \leq \min \left\{x_{1}+c_{1} t, x_{2}+c_{2} t\right\}\right) .
$$

To compute $p_{\mathrm{BB}}(\underline{x}, \underline{c})$, condition on the state of the system at time $t_{\underline{x}}$, i.e., $\bar{B}\left(t_{\underline{x}}\right)$. This random variable is Normally distributed with mean 0 and variance $s_{\underline{x}}:=t_{\underline{x}}\left(1-t_{\underline{x}}\right)$. We get

$$
\begin{aligned}
& p_{\mathrm{BB}}(\underline{x}, \underline{c})= \int_{-\infty}^{y_{\underline{x}}} \frac{1}{\sqrt{2 \pi s_{\underline{x}}}} \exp \left(-\frac{z^{2}}{2 s_{\underline{x}}}\right) p_{\mathrm{BB}}\left(\frac{x_{1}}{\sqrt{t_{\underline{x}}}}, c_{1} \sqrt{t_{\underline{x}}}-\frac{z}{\sqrt{t_{\underline{x}}}}\right) \\
& p_{\mathrm{BB}}\left(\frac{y-z}{\sqrt{1-t_{\underline{x}}}}, c_{2} \sqrt{1-t_{\underline{x}}}+\frac{z}{\sqrt{1-t_{\underline{x}}}}\right) \mathrm{d} z \\
&=\int_{-\infty}^{y_{\underline{x}}} \frac{1}{\sqrt{2 \pi s_{\underline{x}}}} \exp \left(-\frac{z^{2}}{2 s_{\underline{x}}}\right)\left(1-e^{-2 f_{1}(z, \underline{x})}\right)\left(1-e^{-2 f_{2}(z, \underline{x})}\right) \mathrm{d} z,
\end{aligned}
$$

with, by applying (7),

$$
f_{1}(z, \underline{x}):=\frac{x_{1}^{2}-x_{1} z}{t_{\underline{x}}}+c_{1} x_{1} ; \quad f_{2}(z, \underline{x}):=\left(y_{\underline{x}}-z\right)\left(\frac{y_{\underline{x}}}{1-t_{\underline{x}}}+c_{2}\right) .
$$

Tedious calculations (essentially just isolating the square) yield, after having defined $u_{\underline{x}}:=y_{\underline{x}} t_{\underline{x}}+c_{2} s_{\underline{x}}$, and $v_{\underline{x}}:=2 c_{2}^{2} s_{\underline{x}}+4 x_{1} y_{\underline{x}}+4 c_{2} x_{1}\left(1-t_{\underline{x}}\right)+4 c_{2} y_{\underline{x}} t_{\underline{x}}$, and denoting by $\mathcal{N}\left(\mu, \sigma^{2}\right)$ a Normally distributed random variable with mean $\mu$ and variance $\sigma^{2}$,

$$
\int_{-\infty}^{y_{\underline{x}}} \frac{1}{\sqrt{2 \pi s_{\underline{x}}}} \exp \left(-\frac{z^{2}}{2 s_{\underline{x}}}\right) \mathrm{d} z=\mathbb{P}\left(\mathcal{N}\left(0, s_{\underline{x}}\right) \leq y_{\underline{x}}\right) .
$$




$$
\begin{aligned}
& \int_{-\infty}^{y_{\underline{x}}} \frac{1}{\sqrt{2 \pi s_{\underline{x}}}} \exp \left(-\frac{z^{2}}{2 s_{\underline{x}}}-2 f_{1}(z, \underline{x})\right) \mathrm{d} z= \\
& \mathbb{P}\left(\mathcal{N}\left(2 x_{1}\left(1-t_{\underline{x}}\right), s_{\underline{x}}\right) \leq y_{\underline{x}}\right) p_{\mathrm{BB}}\left(x_{1}, c_{1}\right) . \\
& \int_{-\infty}^{y_{\underline{x}}} \frac{1}{\sqrt{2 \pi s_{\underline{x}}}} \exp \left(-\frac{z^{2}}{2 s_{\underline{x}}}-2 f_{2}(z, \underline{x})\right) \mathrm{d} z= \\
& \mathbb{P}\left(\mathcal{N}\left(2 u_{\underline{x}}, s_{\underline{x}}\right) \leq y_{\underline{x}}\right) p_{\mathrm{BB}}\left(y_{x}, c_{2}\right) \exp \left(2 c_{2} u_{\underline{x}}\right) . \\
& \int_{-\infty}^{y_{\underline{x}}} \frac{1}{\sqrt{2 \pi s_{\underline{x}}}} \exp \left(-\frac{z^{2}}{2 s_{\underline{x}}}-2 f_{1}(z, \underline{x})-2 f_{2}(z, \underline{x})\right) \mathrm{d} z= \\
& \mathbb{P}\left(\mathcal{N}\left(2\left(x_{1}\left(1-t_{\underline{x}}\right)+u_{\underline{x}}\right), s_{\underline{x}}\right) \leq y_{\underline{x}}\right) p_{\mathrm{BB}}^{-}\left(x_{1}, c_{1}\right) p_{\mathrm{BB}}^{-}\left(y_{x}, c_{2}\right) \exp \left(v_{\underline{x}}\right) .
\end{aligned}
$$

We have proved the following proposition.

Proposition 4.1 If $t_{\underline{x}} \geq 1$ then $p_{\mathrm{BB}}(\underline{x}, \underline{c})=p_{\mathrm{BB}}\left(x_{1}, c_{1}\right)$. If $t_{\underline{x}}<1$, then $p_{\mathrm{BB}}(\underline{x}, \underline{c})$ equals

$$
\begin{aligned}
& \mathbb{P}\left(\mathcal{N}\left(0, s_{\underline{x}}\right) \leq y_{\underline{x}}\right)-\mathbb{P}\left(\mathcal{N}\left(2 x_{1}\left(1-t_{\underline{x}}\right), s_{\underline{x}}\right) \leq y_{\underline{x}}\right) p_{\mathrm{BB}}\left(x_{1}, c_{1}\right)- \\
& \mathbb{P}\left(\mathcal{N}\left(2 u_{\underline{x}}, s_{\underline{x}}\right) \leq y_{\underline{x}}\right) p_{\mathrm{BB}}^{-}\left(y_{x}, c_{2}\right) \exp \left(2 c_{2} u_{\underline{x}}\right)+ \\
& \mathbb{P}\left(\mathcal{N}\left(2\left(x_{1}\left(1-t_{\underline{x}}\right)+u_{\underline{x}}\right), s_{\underline{x}}\right) \leq y_{\underline{x}}\right) p_{\mathrm{BB}}\left(x_{1}, c_{1}\right) p_{\mathrm{BB}}\left(y_{x}, c_{2}\right) \exp \left(v_{\underline{x}}\right) .
\end{aligned}
$$

It has been argued, see e.g. Roberts et al. [13, Section 15.2.2] and [11, Section III.D], that if $N$ arrivals are distributed uniformly on $[0, D)$, then $A(t D)-t N$ is close to $\sqrt{N} \bar{B}(t)$, for $t \in[0,1)$. This leads to the following approximation, which is particularly accurate under heavy-traffic conditions:

Approximation 4.2 With $p_{\mathrm{BB}}(\cdot, \cdot)$ as above,

$$
p_{\mathrm{t}}(\underline{x}, \underline{c} \mid N, D) \approx p_{\mathrm{BB}}\left(\left(\frac{x_{1}}{\sqrt{N}}, \frac{x_{2}}{\sqrt{N}}\right),\left(c_{1} \frac{D}{\sqrt{N}}-\sqrt{N}, c_{2} \frac{D}{\sqrt{N}}-\sqrt{N}\right)\right) .
$$




\subsection{Approximations for the tandem queue with Poisson arrivals}

A standard result from the theory of Brownian motions is that, for $x, c \geq 0$,

$$
q_{\mathrm{BM}}(x, c):=\mathbb{P}(\forall t \in[0, \infty): B(t) \leq x+c t)=1-q_{\overline{\mathrm{BM}}}(x, c),
$$

with $q_{\overline{\mathrm{BM}}}^{-}(x, c):=\exp (-2 x c)$, see $[13$, Eq. (15.1.4)]. Now define

$$
q_{\mathrm{BM}}(\underline{x}, \underline{c}):=\mathbb{P}\left(\forall t \in[0, \infty): B(t) \leq \min \left\{x_{1}+c_{1} t, x_{2}+c_{2} t\right\}\right) .
$$

To compute $q_{\mathrm{BM}}(\underline{x}, \underline{c})$, again condition on the state of the system at time $t_{\underline{x}}$, i.e., $B\left(t_{\underline{x}}\right)$. This random variable is Normally distributed with mean 0 and variance $t_{\underline{x}}$. We get

$$
\begin{gathered}
q_{\mathrm{BM}}(\underline{x}, \underline{c})=\int_{-\infty}^{y_{\underline{x}}} \frac{1}{\sqrt{2 \pi t_{\underline{x}}}} \exp \left(-\frac{z^{2}}{2 t_{\underline{x}}}\right) p_{\mathrm{BB}}\left(\frac{x_{1}}{\sqrt{t_{\underline{x}}}}, c_{1} \sqrt{t_{\underline{x}}}-\frac{z}{\sqrt{t_{\underline{x}}}}\right) \\
q_{\mathrm{BM}}\left(y_{\underline{x}}-z, c_{2}\right) \mathrm{d} z
\end{gathered}
$$

This eventually leads to the following result.

Proposition $4.3 q_{\mathrm{BM}}(\underline{x}, \underline{c})$ equals

$$
\begin{aligned}
& \mathbb{P}\left(\mathcal{N}\left(0, t_{\underline{x}}\right) \leq y_{\underline{x}}\right)-\mathbb{P}\left(\mathcal{N}\left(x_{1}, t_{\underline{x}}\right) \leq y_{\underline{x}}\right) q_{\mathrm{BM}}^{\overline{\mathrm{BM}}}\left(x_{1}, c_{1}\right)- \\
& \mathbb{P}\left(\mathcal{N}\left(2 c_{2} t_{\underline{x}}, t_{\underline{x}}\right) \leq y_{\underline{x}}\right) q_{\overline{\mathrm{BM}}}\left(y_{\underline{x}}, c_{2}\right) \exp \left(2 c_{2}^{2} t_{\underline{x}}\right)+ \\
& \mathbb{P}\left(\mathcal{N}\left(x_{1}+2 c_{2} t_{\underline{x}}, t_{\underline{x}}\right) \leq y_{\underline{x}}\right) q_{\mathrm{BM}}^{-}\left(x_{1}, c_{1}\right) q_{\mathrm{BM}}^{-}\left(y_{\underline{x}}, c_{2}\right) \exp \left(2 c_{2}^{2} t_{\underline{x}}+4 c_{2} x_{1}\right) .
\end{aligned}
$$

Now look at the tandem system with arrivals according to a Poisson process of rate $\lambda$. With $A(t)-\lambda t$ being close to $\sqrt{\lambda} B(t)$, we propose the following approximation.

Approximation 4.4 With $q_{\mathrm{BM}}(\cdot, \cdot)$ as above,

$$
q_{\mathrm{t}}(\underline{x}, \underline{c} \mid \lambda) \approx q_{\mathrm{BM}}\left(\left(\frac{x_{1}}{\sqrt{\lambda}}, \frac{x_{2}}{\sqrt{\lambda}}\right),\left(\frac{c_{1}}{\sqrt{\lambda}}-\sqrt{\lambda}, \frac{c_{1}}{\sqrt{\lambda}}-\sqrt{\lambda}\right)\right) .
$$




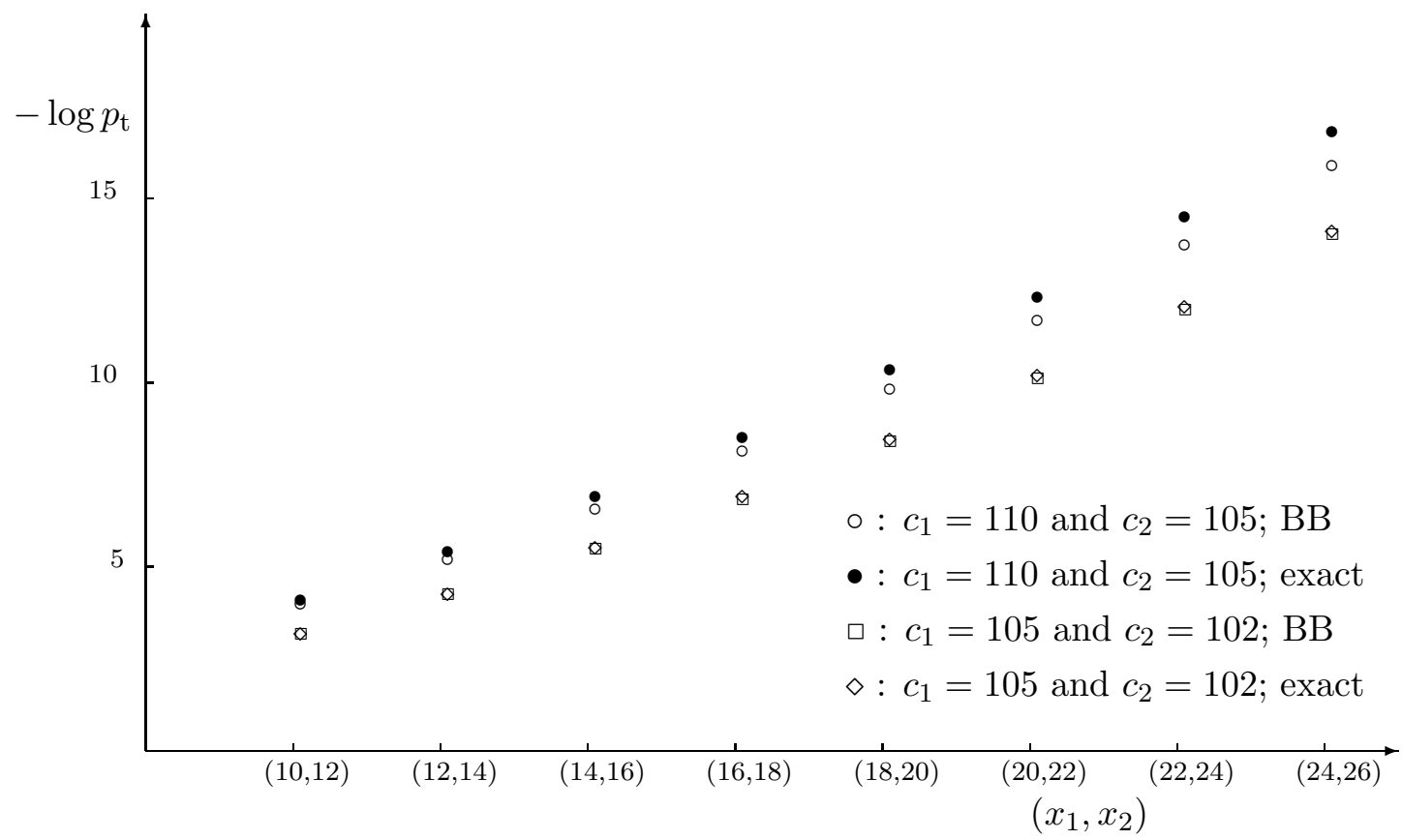

Figure 3: Probability $p_{\mathrm{t}}(\underline{x}, \underline{c} \mid N, D)$, as a function of $\left(x_{1}, x_{2}\right)$, for $N=100$.

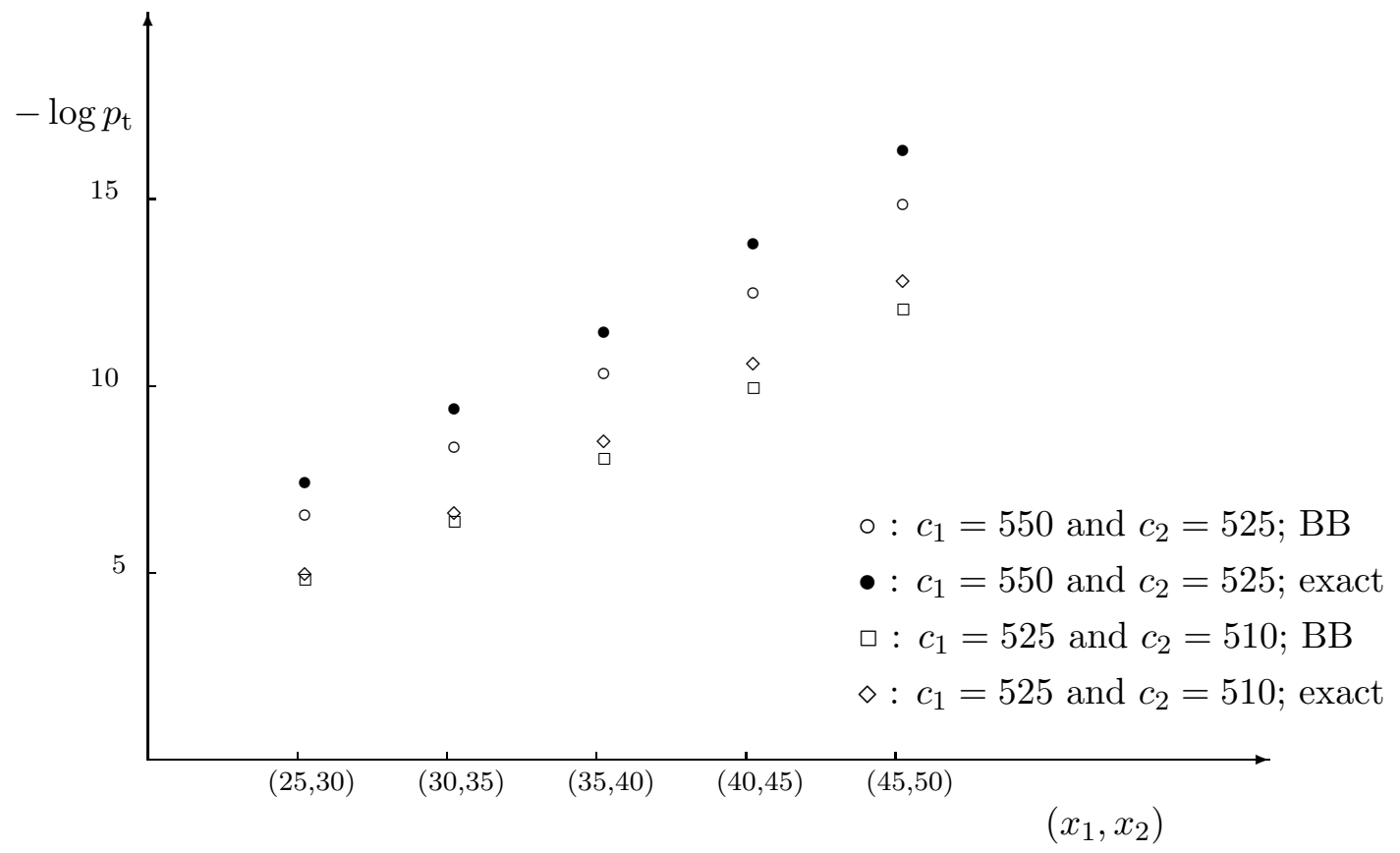

Figure 4: Probability $p_{\mathrm{t}}(\underline{x}, \underline{c} \mid N, D)$, as a function of $\left(x_{1}, x_{2}\right)$, for $N=500$. 


\begin{tabular}{|c|c|c|c|c|}
\hline$\left(x_{1}, x_{2}\right)$ & \begin{tabular}{l|l|l}
$p_{\mathrm{t}}(\underline{x}, \underline{c}$ & $N, D)$
\end{tabular} & $\mathrm{BB}$ & $\begin{array}{l}p_{\mathrm{t}}(\underline{x}, \underline{c} \mid N, D) \\
\end{array}$ & $\mathrm{BB}$ \\
\hline & \multicolumn{2}{|c|}{$c_{1}=110 ; c_{2}=105$} & \multicolumn{2}{|c|}{$c_{1}=105 ; c_{2}=102$} \\
\hline$(10,12)$ & $2.2 \cdot 10^{-2}$ & $2.0 \cdot 10^{-2}$ & $5.0 \cdot 10^{-2}$ & $5.0 \cdot 10^{-2}$ \\
\hline$(12,14)$ & $6.6 \cdot 10^{-3}$ & $5.4 \cdot 10^{-3}$ & $1.7 \cdot 10^{-2}$ & $1.7 \cdot 10^{-2}$ \\
\hline$(14,16)$ & $1.7 \cdot 10^{-3}$ & $1.2 \cdot 10^{-3}$ & $5.0 \cdot 10^{-3}$ & $4.9 \cdot 10^{-3}$ \\
\hline$(16,18)$ & $3.5 \cdot 10^{-4}$ & $2.4 \cdot 10^{-4}$ & $1.3 \cdot 10^{-3}$ & $1.2 \cdot 10^{-3}$ \\
\hline$(18,20)$ & $6.5 \cdot 10^{-5}$ & $3.8 \cdot 10^{-5}$ & $2.7 \cdot 10^{-4}$ & $2.5 \cdot 10^{-4}$ \\
\hline$(20,22)$ & $1.0 \cdot 10^{-6}$ & $5.3 \cdot 10^{-6}$ & $4.9 \cdot 10^{-5}$ & $4.5 \cdot 10^{-5}$ \\
\hline$(22,24)$ & $1.3 \cdot 10^{-6}$ & $6.1 \cdot 10^{-7}$ & $7.5 \cdot 10^{-6}$ & $6.9 \cdot 10^{-6}$ \\
\hline$(24,26)$ & $1.5 \cdot 10^{-7}$ & $6.0 \cdot 10^{-8}$ & $9.7 \cdot 10^{-7}$ & $9.0 \cdot 10^{-7}$ \\
\hline
\end{tabular}

Table 1: Exact results vs. Brownian bridge, $N=100$

\begin{tabular}{|c|c|c|c|c|}
\hline$\left(x_{1}, x_{2}\right)$ & $p_{\mathrm{t}}(\underline{x}, \underline{c} \mid N, D)$ & BB & $\begin{array}{l}p_{\mathrm{t}}(\underline{x}, \underline{c} \mid N, D) \\
\end{array}$ & BB \\
\hline & \multicolumn{2}{|c|}{$c_{1}=550 ; c_{2}=525$} & \multicolumn{2}{|c|}{$c_{1}=525 ; c_{2}=510$} \\
\hline$(25,30)$ & $1.7 \cdot 10^{-3}$ & $7.2 \cdot 10^{-4}$ & $9.6 \cdot 10^{-3}$ & $8.2 \cdot 10^{-3}$ \\
\hline$(30,35)$ & $2.8 \cdot 10^{-4}$ & $1.0 \cdot 10^{-4}$ & $2.1 \cdot 10^{-3}$ & $1.6 \cdot 10^{-3}$ \\
\hline$(35,40)$ & $3.9 \cdot 10^{-5}$ & $1.3 \cdot 10^{-5}$ & $3.8 \cdot 10^{-4}$ & $2.4 \cdot 10^{-4}$ \\
\hline$(40,45)$ & $4.5 \cdot 10^{-6}$ & $1.2 \cdot 10^{-6}$ & $5.7 \cdot 10^{-5}$ & $3.0 \cdot 10^{-5}$ \\
\hline$(45,50)$ & $4.3 \cdot 10^{-7}$ & $1.0 \cdot 10^{-7}$ & $7.0 \cdot 10^{-6}$ & $3.3 \cdot 10^{-6}$ \\
\hline
\end{tabular}

Table 2: Exact results vs. Brownian bridge, $N=500$

\subsection{Numerical experiments}

In this subsection we compare the exact results for the tandem fed by periodic input with the Brownian bridge approximation of Section 4.1. In our example we choose $D=1$. The examples are chosen such that we are in the (interesting) regime $t_{\underline{x}}<D$.

The results given in Tables 1-2 and Figures 3-4 illustrate the accuracy of the Brownian bridge approximation. As argued before, the approximation becomes more accurate when the load increases, as is clear from the table this was already known for the single queue, see [13, Table 15.2.1]. In the numerical experiments we performed, it turned out that the Brownian bridge estimate was usually slightly optimistic. 


\section{CONCLUDING REMARKS}

In this section we give a number of comments on the analysis, and identify directions for further research.

- In our analysis of the tandem queue, we 'do as if traffic leaves the first queue as fluid' - as a consequence $\bar{Q}$ behaves as a single queue emptied at rate $c_{2}$. In reality however, a packet moves to the second queue only when its service is completed. We expect that this effect is of minor impact (particularly in situations with many sources, such as the scenarios evaluated in the numerical experiments). It is, however, possible to adapt out formulas to incorporate the 'packet nature' of the output stream of the first queue, by adding an additional term to $\bar{Q}$; it turns out that there is a 1-to-1 mapping between the system with fluid output and the system with packet output, that provides this correction term directly.

A similar remark applies to the priority system. There we tacitly assumed that the service of a low-priority packet can be interrupted.

- There are many interesting directions for further research. Suppose for instance that queue 1 has $N$ inputs, of which $i$ leave the system after the first queue, and suppose that these are replaced at the second queue by $i$ new i.i.d. periodic streams (or perhaps $j \neq i$ new streams). This model is more realistic in a network setting, but much harder to analyze.

Another interesting extension could incorporate a flow level on top of the packet level. One could for instance consider the queue with periodic arrivals $(N \cdot \mathrm{D} / \mathrm{D} / 1$ type), but with the number of flows fluctuating according to the Erlang loss model. In the model with a fixed number of flows there is no jitter: the output streams are purely periodical; however, the model with a fluctuating number of flows does incur jitter: during its duration, a flow sees a variable queueing delay. 


\section{References}

[1] V. BEnEš. General stochastic processes in the theory of queues. Addison Wesley, Reading MA, 1963.

[2] A. Bhargava, P. Humblet, and M. HLUCHYJ. Queueing analysis of continuous bitstream transport in packet networks. Proceedings IEEE Globecom, 1989.

[3] P. Boyer, A. Dupuis, A. Gravey, and J.-M. Pitie. The output process of the single server queue with periodic arrival process and deterministic service time. In: Modelling and performance evaluation methodology: proceedings of the international seminar, Paris, France, F. Baccelli and G. Fayolle, Eds. Lecture Notes in Control and Information Sciences, Vol. 60. Springer, Berlin, 1984.

[4] A. Dempster. Generalized $D_{n}^{+}$statistics. Annals of Mathematical Statistics, 30: 593 - 597, 1959.

[5] A.E. EckberG, JR. The single server queue with periodic arrival process and deterministic service time. IEEE Transactions on Communications, 27: 556 - 562, 1979.

[6] A. Gravey. Temps d'attente et nombre de clients dans une file $\mathrm{nD} / \mathrm{D} / 1$. Annales d'institut H. Poincaré - Probabilités et Statistiques, 20: $53-73,1984$.

[7] B. HAJEK. A queue with periodic arrivals and constant service rate. In: Probability, Statistics and Optimisation - a Tribute to Peter Whittle. F.P. Kelly, ed.; John Wiley and Sons, 147 - 158, 1994.

[8] P. Humblet, A. Bhargava, and M. HLuCHYJ. Ballot theorems applied to the transient analysis of $\mathrm{nD} / \mathrm{D} / 1$ queues. IEEE/ACM Transactions on Networking, 1: 81 - 95, 1993.

[9] K. Iida, T. Takine, H. Sunahara, and Y. OIE. Delay analysis for CBR traffic under static priority scheduling. IEEE/ACM Transactions on Networking, 9: 177 - 185, 2001.

[10] M. Mandjes, K. VAn der Wal, R. KooiJ, and H. BastiaAnsen. End-to-end delay models for interactive services on a large-scale IP network. Proceedings 7 th IFIP workshop on modeling and evaluation of ATM/IP networks, Antwerp, Belgium, 1999.

[11] I. Norros, J. Roberts, A. Simonian, and J. Virtamo. The superposition of variable bit rate sources in an ATM multiplexer. IEEE Journal on Selected Areas in Communications, 9: $378-387,1991$.

[12] R. PykE. The supremum and infimum of the Poisson process. Annals of Mathematical Statistics, 30: 568 - 576, 1959.

[13] J. Roberts, U. Mocci, and J. Virtamo. Broadband network teletraffic. Final report of action COST 242. Springer, Berlin, 1996.

[14] J. Roberts and J. Virtamo. The superposition of periodic cell arrival streams in an ATM multiplexer. IEEE Transactions on Communications, 39: 298 - 303, 1991.

[15] I. Rubin. Path delays in communication networks. Applied Mathematics and Optimization, 1: $193-221,1974$.

[16] B. Sengupta. A queue with superposition of arrival streams with an application to packet voice technology. Proceedings Performance 1990, 53 - 60, 1990.

[17] M. Shalmon and M. Kaplan. A tandem network of queues with deterministic service and intermediate arrivals. Operations Research, 32 : $753-773,1984$.

[18] G. Shorack and J. Wellner. Empirical processes with applications to Statistics. Wiley, New York, 1967.

[19] L. TAKÁCS. Combinatorial methods in the theory of stochastic processes. Wiley, New York NY, 1967.

[20] H. TAKagi. Queueing Analysis. A foundation of Performance Evaluation. Vol. I: Vacation and Priority Systems, Part 1. North-Holland, Amsterdam, 1991.

[21] J. Virtamo. Idle and busy period distributions of an infinite capacity $N^{*} \mathrm{D} / \mathrm{D} / 1$ queue. Proceedings ITC 14, 453 - 459, 1994. 
[22] J. Virtamo and J. Roberts. Evaluating buffer requirements in an ATM multiplexer. Proceedings IEEE Globecom, 1989.

[23] K. Van der Wal, M. Mandjes, and H. BASTIAANSEN. Delay performance analysis of the new internet services with guaranteed QoS. Proceedings of the IEEE, 85: 1947 - 1957, 1997. 